\title{
Ratiometric detection of enzyme turnover and flavin reduction using rare-earth upconverting phosphors $\dagger$
}

5265

Received 3rd February 2014,

Accepted 7th February 2014

DOI: 10.1039/c4dt00356j

www.rsc.org/dalton
Peter Harvey, ${ }^{a, b, c}$ Chloë Oakland, ${ }^{a, b, c}$ Max D. Driscoll, ${ }^{b}$ Sam Hay ${ }^{\star b, c}$ and Louise S. Natrajan*a,c
$\mathrm{Gd}_{4} \mathrm{O}_{2} \mathrm{~S}: \mathrm{Yb}: \mathrm{Tm}$ rare-earth upconversion phosphors have been utilised to monitor the redox behaviour of flavin mononucleotide and report on the turnover of a flavo-protein, (pentaerythritol tetranitrate reductase). The presence of two bands separated by over $300 \mathrm{~nm}$ in the UCP emission spectra allows ratiometric signalling of these processes with high sensitivity.

Rare-earth upconverting phosphors (UCPs) are a rapidly emerging class of (nano)particles with potential application in sensing and imaging, optical memory, security, and photodynamic therapy. ${ }^{1}$ Upon excitation with near-infrared (NIR) light, UCPs exhibit efficient photoluminescence in the visible spectrum due to photon upconversion (UC). The type and efficiency of the UC process, in addition to the size and phase of the UCPs, can be tuned by doping the UCPs with varying levels of certain lanthanides ${ }^{2}$ and involves a non-linear multiphoton absorption process. This mechanism is based on sequential absorption and energy transfer of NIR photons, leading to the population of real, metastable excited states (here of $\mu$ s order), differing to the virtual excited state involved in two-photon absorption (Fig. S1†). ${ }^{3}$ Due to this UC behaviour, probes based on these UCPs exhibit no auto-fluorescence (i.e. noise) in biological media and negligible photobleaching. ${ }^{4}$ These advantages over conventional fluorophores, combined with relatively low associated toxicities, ${ }^{5}$ has led to a particular focus on bioimaging, with relevant applications in in vitro studies. ${ }^{6}$ UCPs have also be applied as optical chemosensors, with systems developed for $\mathrm{pH}, \mathrm{O}_{2}, \mathrm{Hg}$ (II), $\mathrm{Cu}(\mathrm{II})$, and $\mathrm{Fe}(\mathrm{III})$ recognition, ${ }^{7}$ amongst others. The synthesis, characterisation,

\footnotetext{
${ }^{a}$ School of Chemistry, The University of Manchester, Oxford Road, Manchester, M13 9PL, UK. E-mail: louise.natrajan@manchester.ac.uk; Tel: +44 (0)161 2751426 ${ }^{b}$ Manchester Institute of Biotechnology, The University of Manchester, 131 Princess Street, Manchester, M1 7DN, UK. E-mail: Sam.Hay@manchester.ac.uk; Tel: +44 (0)161306 5141

${ }^{c}$ The Photon Science Institute, The University of Manchester, Oxford Road, Manchester, M13 9PL, UK

$\dagger$ Electronic supplementary information (ESI) available: Synthetic and experimental details. See DOI: 10.1039/c4dt00356j
}

and properties of these UCPs have been well summarised in a number of key reviews. ${ }^{8}$

The unparalleled substrate selectivity and sub-nM substrate affinity of enzymes offers a sensitive and selective method of sensing biomolecules. For enzymes that contain chromophoric substrates/intrinsic cofactors, it should be feasible to induce Förster resonance energy transfer (FRET) ${ }^{9}$ between suitable UCPs (donor) and enzyme/substrate chromophores (acceptor). As the spectral absorption properties of enzyme chromophores typically change during redox enzyme turnover, the FRET efficiency will be sensitive to the presence of enzyme substrates. This is the proposed basis for a new class of luminescent enzyme-based biosensor, with proof-of-principle studies described herein.

Pentaerythritol tetranitrate reductase (PETNR) belongs to the old yellow enzyme (OYE) family of $\mathrm{NAD}(\mathrm{P}) \mathrm{H}$ (reduced Nicotinamide Adenine Dinucleotide (Phosphate)) dependant enzymes. ${ }^{10}$ This family of flavoproteins, which are capable of accommodating a wide-range of substrates,,${ }^{11}$ have played a significant role in the development of enzymology. PETNR was first isolated from explosives-contaminated soil ${ }^{12}$ and catalyses the reduction of unsaturated activated alkenes (e.g. 2-cyclohexen-1-one), aromatic and aliphatic nitro-containing compounds (e.g. PETN), and aromatic ring systems (e.g. TNT) ${ }^{13}$ Despite the considerable amount of work carried out on OYEs, their physiological role is still a matter of contention.

In this study, we present a novel application of rare-earth UCPs to probe the redox behaviour of PETNR, utilising FRET between the emission bands of the UCP and the enzyme absorbance band. Due to a variation in the absorbance profile of the flavin core of the enzyme upon reduction/oxidation, the FRET between the two can effectively be turned 'on' or 'off' by changing the redox state of PETNR (Fig. 1). To the best of our knowledge, this is the first time such upconversion particles have been used to probe protein function.

The UCPs used in this study (PTIR 475) were obtained from Phosphor Technology Ltd and directly suspended in aqueous TRIS buffer solution ( $\mathrm{pH} 7$ ). Following continuous wave (CW) 


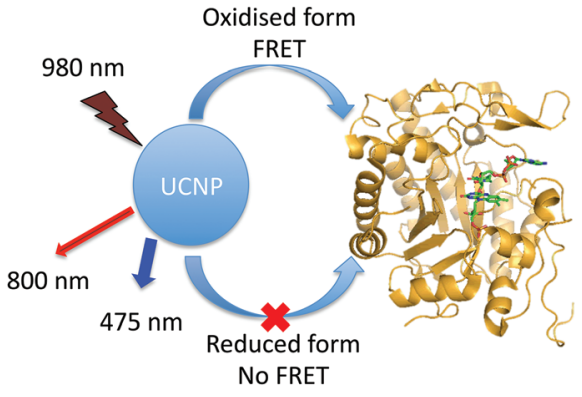

Fig. 1 Schematic illustration of 'on-off' FRET process based on UCPs and PETNR. Reduction of the enzyme inhibits FRET and alters the intensity of the $475 \mathrm{~nm}$ band in the UCP emission spectrum.

$980 \mathrm{~nm}$ excitation, these UCPs give dual-emission bands in the blue and near IR regions of the electromagnetic spectrum.

The PTIR 475 emission bands centred at $475 \mathrm{~nm}$ and $800 \mathrm{~nm}$ can be assigned to ${ }^{1} \mathrm{G}_{4} \rightarrow{ }^{3} \mathrm{H}_{6}$ and ${ }^{3} \mathrm{H}_{4} \rightarrow{ }^{3} \mathrm{H}_{6}$ transitions of $\mathrm{Tm}^{3+}$, respectively (Fig. $\mathrm{S} 1 \dagger$ ). ${ }^{14}$ The separation of over $300 \mathrm{~nm}$ between these transitions can be utilised to great effect for determining relative concentrations by normalising the band at $800 \mathrm{~nm}$ (also known as ratiometric analysis), a technique that has been previously applied for related lanthanide-based systems. ${ }^{15}$ As the intensity of the NIR emission at $800 \mathrm{~nm}$ is independent of added flavin/enzyme concentration, measuring responses as a variation in the ratio between these two emission bands negates any probe concentration dependence. ${ }^{16}$ This ratiometric analysis is particularly important for such systems that exist as a dispersion in solution with variable concentration per scan. All experiments were carried out in $100 \mathrm{mM}$ TRIS buffer, $10 \mathrm{mM} \mathrm{NaCl}, \mathrm{pH} 7$.

Initial studies focussed on the energy transfer between the UCPs and flavin mononucleotide (FMN) free in solution. The flavin absorption band centred at $\sim 460 \mathrm{~nm}$ has good spectral overlap with the $475 \mathrm{~nm}$ UNCP emission band (Fig. 2), allowing FRET to occur from the UNCP to FMN. Upon two-electron reduction, the $460 \mathrm{~nm}$ absorption of FMN is lost and the solution becomes colourless (Fig. S8 $\dagger$ ), thus preventing such FRET. This variable FRET behaviour should result in a decrease in the $475 \mathrm{~nm}$ emission band of the UCP upon FMN addition, followed by full return of this emission upon reduction of the

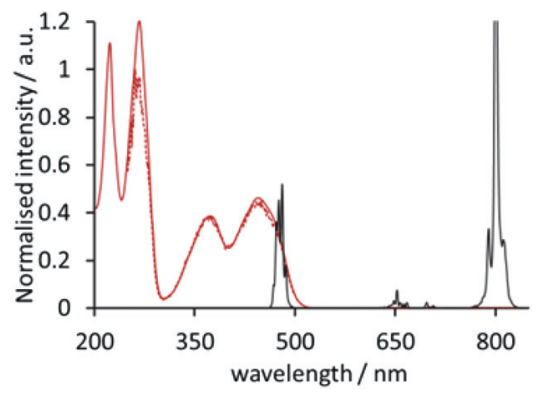

Fig. 2 Overlay of FMN absorption (solid red line), PETNR absorption (dashed red line), and PTIR 475 emission (black line) spectra with relative intensities scaled for comparison.

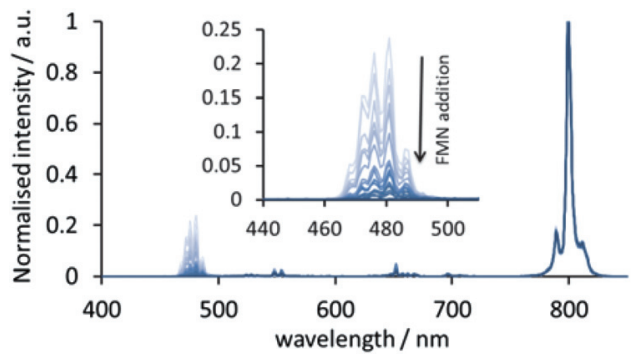

Fig. 3 Variation of UCP emission spectra upon incremental addition of FMN, with decrease in $475 \mathrm{~nm}$ emission expanded in inset. The emission band at $800 \mathrm{~nm}$ is unaffected by increased flavin concentration, enabling for ratiometric analysis of the data.

FMN. As the UCPs are excited with NIR laser at $980 \mathrm{~nm}$, direct FMN excitation is avoided and background noise is eliminated.

In order to further test this FRET process, an aqueous solution of UCPs $(0.01 \mathrm{wt} \%)$ was irradiated by CW $980 \mathrm{~nm}$ diode laser excitation and the emission spectrum recorded. To this solution was added FMN ( $2 \mathrm{mM}$ aqueous solution) in incremental additions, from 10 to $500 \mu \mathrm{M}$, resulting in a gradual decrease of the $475 \mathrm{~nm}$ UCP emission band (Fig. 3). While the absorption of the FMN may cause significant inner filter effects during visible excitation of these samples, this is negated by the NIR excitation of the UCPs. Also, by analysing the total area of the $475 \mathrm{~nm}$ band as a ratio of the area of the $800 \mathrm{~nm}$ emission band, which is unaffected by the addition of FMN, slight fluctuations in emission intensity per measurement due to the UCPs existing as a dispersion in solution can be avoided. A Stern-Volmer analysis (see ESI $\dagger$ ) of this ratio as a function of FMN concentration displays multiple linear regions, suggesting the occurrence of both static and dynamic quenching mechanisms, the relative contributions of which change as a function of increasing concentration (Fig. S2 $\dagger$ ). The existence of additional non-radiative quenching pathways that serve to decrease the emission intensity of the $\mathrm{Tm}^{3+}$ based emission by the introduction of proximal $\mathrm{O}-\mathrm{H}$ and $\mathrm{C}-\mathrm{H}$ oscillators may also affect the Stern-Volmer analyses.

In order to mimic the behaviour of protein-bound FMN during enzyme turnover, it is necessary to observe the FRET process in the presence of an FMN-reducing agent. In order to investigate this, a solution containing PTIR 475 phosphors $(0.01 \mathrm{wt} \%)$ and FMN $(100 \mu \mathrm{M})$ in TRIS buffer was prepared and deoxygenated by bubbling with $\mathrm{N}_{2}$. To this solution was added sodium dithionite $(600 \mu \mathrm{M})$, also prepared as a separate anaerobic solution in TRIS buffer. As anticipated, upon reduction of the FMN, the FRET process between the flavin and UCPs is effectively turned off and the original UCP emission spectrum is restored (Fig. S3a $\dagger$ ). The spectral effects of this reduction can also be observed physically, with a loss of the deep yellow colour of the flavin in solution. The FRET process can be restored simply by shaking the sample in air to reoxidise the FMN. The reduction and subsequent oxidation can be cycled multiple times $(>3)$ with no apparent negative effect to either flavin or phosphors. These processes can also 

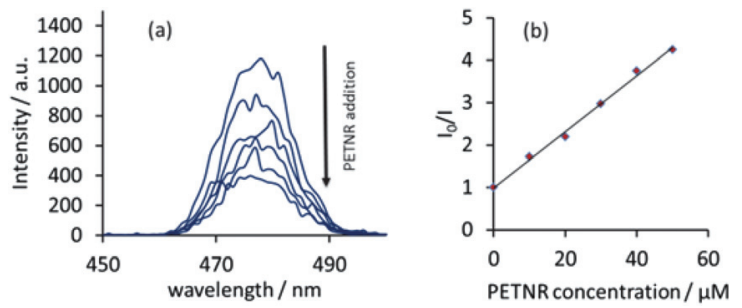

Fig. 4 (a) Variation of UCP emission spectra upon incremental addition of PETNR, with only the $475 \mathrm{~nm}$ emission band of interest shown; (b) Stern-Volmer analysis of the 475 to $800 \mathrm{~nm}$ emission intensity ratio as a function of PETNR concentration, where $I_{0}$ is the emission intensity before enzyme addition and $I$ is the intensity upon $\times \mu \mathrm{M}$ PETNR addition.

be analysed experimentally by observing direct flavin emission upon $475 \mathrm{~nm}$ irradiation (Fig. S3b†).

These promising studies with FMN led to investigations into the interactions between the full enzyme system and UCPs. PETNR was prepared following literature procedures. ${ }^{17}$ The FRET process between the two systems was achieved by irradiating a solution of UCPs $(0.01 \mathrm{wt} \%)$ with $\mathrm{CW} 980 \mathrm{~nm}$ excitation, followed by incremental addition of PETNR. A similar response was observed to that with flavin, with a reduction in the $475 \mathrm{~nm}$ UCP emission band with increasing PETNR concentration (Fig. 4). Comparison of the SternVolmer analyses over the relevant concentration range $(0-50 \mu \mathrm{M})$ suggests electrostatic binding in solution is much stronger in the enzyme-UCP system than in the FMN-UCP system (Fig. S4 $\dagger$ ).

FRET-induced flavin emission can also be observed upon addition of the enzyme (Fig. S5 $\dagger$ ). However, due to the broad nature of the flavin emission in comparison the narrow, linelike luminescence of the UCPs, this emission appears very weak in comparison, highlighting an advantage of using lanthanide-based emission over standard organic chromophores.

Due to the weak nature of this FRET-induced flavin emission band, FRET efficiency calculations (discussed in detail for related systems elsewhere ${ }^{18}$ using changes in the acceptor excited state lifetimes proved unfeasible. No significant change in the UCP emission lifetime $\left(\tau_{475} \mathrm{~nm}=88 \pm 1 \mu \mathrm{s}\right.$, $\tau_{800 \mathrm{~nm}}=172 \pm 1 \mu \mathrm{s}$ ) was observed upon the addition of $\mu \mathrm{M}$ aliquots of either FMN or PETNR. Combined with the results from the Stern-Volmer analysis, these data suggest predominantly static quenching is occurring in this system, particularly when PETNR or FMN is at low concentration. UV-vis absorption spectra were unsuitable for monitoring this quenching due to the high level of scatter caused by the UCPs in solution.

Next, to model enzyme turnover, reduction of the protein was investigated with the addition of NADPH under anaerobic conditions (Fig. 5). As with the sodium dithionite reduction of FMN, an initial reduction in the $475 \mathrm{~nm}$ UCP emission band is observed upon addition of $100 \mu \mathrm{M}$ PETNR. As the enzyme is reduced by NADPH $(200 \mu \mathrm{M})$, the FRET process between PETNR and the UCPs is far less efficient, resulting in almost full recovery of the $475 \mathrm{~nm}$ emission. By exposing the sample

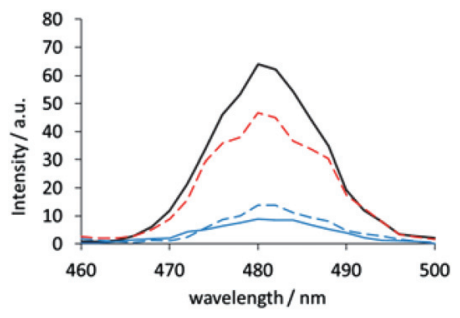

Fig. 5 PTIR 475 UCP emission $\left(\lambda_{\text {exc }}=980 \mathrm{~nm}\right.$ ) in anaerobic solution before (black line) and after (blue line) addition of $100 \mu \mathrm{M}$ PETNR, followed by reduction of the enzyme with $200 \mu \mathrm{M}$ NADPH (red dashed line) and subsequent reoxygenation in air (blue dashed line).

to air, the enzyme is reoxidised and the FRET process is restored. Again, this behaviour can be confirmed by observing direct enzyme emission (Fig. S6 $\dagger$ ). These redox states can be repetitively cycled ( $>5$ times) with no apparent degradation of either the UCPs or the enzyme, indicating the stable reversibility of the system. Incremental addition of NADPH under anaerobic conditions can also be measured as a ratiometric response (Fig. S9†), establishing the basis for the system to act as a responsive probe for various species in solution.

\section{Conclusions}

This study indicates a proof-of-principle basis for the use of UCPs to report on the function of biological systems; in this case the redox state of a flavoenzyme. As the emission profile of the UCPs can be tuned by altering the rare-earth dopant concentrations, this technique can be expanded to monitor other biological chromophores. Investigations are underway to improve concentration limits by covalently binding UCPs to biological compounds, in order to develop functional in vitro bioassay systems. This work establishes the basis for the development of a new class of responsive luminescent probe systems that combine the advantages of UCPs (NIR excitation, no autofluorescence, etc.) with the high sensitivity and selectivity of enzymes for ratiometric detection of enzyme-substrate activity e.g. during substrate and/or enzyme variant screens in biocatalysis.

We thank Phosphor Technology Ltd for the UCPs, Prof. Nigel Scrutton for the PETNR expression plasmid and the EPSRC (LN, PH, CO) and BBSRC (SH, MD) for financial support. SH is a David Phillips Fellow and LN is a Career Acceleration Fellow.

\section{Notes and references}

1 L. Xiong, Z. Chen, M. Yu, F. Li, C. Liu and C. Huang, Biomaterials, 2009, 30, 5592; C. Carling, J. Boyer and N. R. Branda, J. Am. Chem. Soc., 2009, 131, 10838; W. J. Kim, M. Nyk and P. N. Prasad, Nanotechnology, 2009, 20, 185301; P. Zhang, W. Steelant, M. Kumar and M. Scholfield, J. Am. Chem. Soc., 2007, 129, 4526. 
2 F. Wang, Y. Han, C. S. Lim, Y. Lu, J. Wang, J. Xu, H. Chen, C. Zhang, M. Hong and X. Liu, Nature, 2010, 463, 1061.

3 L. S. Natrajan, A. Toulmin, A. Chew and S. W. Magennis, Dalton Trans., 2010, 39, 10837.

4 F. Wang, D. Banerjee, Y. Liu, X. Chen and X. Liu, Analyst, 2010, 135, 1839.

5 L. Cheng, K. Yang, M. Shao, X. Lu and Z. Liu, Nanomedicine, 2011, 6, 1327.

6 S. Sivakumar, P. R. Diamente and F. C. J. M. van Veggel, Chem.-Eur. J., 2006, 12, 5878; L. Wang, R. Yan, Z. Huo, L. Wang, J. Zeng, J. Bao, X. Wang, Q. Peng and Y. Li, Angew. Chem., Int. Ed., 2005, 44, 6054.

7 L. Sun, H. Peng, M. I. J. Stich, D. Achatz and O. S. Wolfbeis, Chem. Commun., 2009, 5000; D. E. Achatz, R. J. Meier, L. H. Fischer and O. S. Wolfbeis, Angew. Chem., Int. Ed., 2011, 50, 260; Q. Liu, J. Peng, L. Sun and F. Li, ACS Nano, 2011, 5, 8040; J. Zhang, B. Li, L. Zhang and H. Jiang, Chem. Commun., 2012, 48, 4860; Y. Ding, H. Zhu, X. Zhang, J. Zhu and C. Burda, Chem. Commun., 2013, 49, 7797.

8 M. Haase and H. Schäfer, Angew. Chem., Int. Ed., 2011, 50, 5808; C. Li and J. Lin, J. Mater. Chem., 2010, 20, 6831; J. F. Suyver, A. Aebischer, D. Biner, P. Gerner, J. Grimm, S. Heer, K. W. Krämer, C. Reinhard and H. U. Güdel, Opt. Mater., 2005, 27, 1111.

9 H. N. Barnhill, S. Claudel-Gillet, R. Ziessel, L. J. Charbonnière and Q. Wang, J. Am. Chem. Soc., 2007, 129, 7799.
10 R. E. Williams and N. C. Bruce, Microbiology, 2002, 148, 1607.

11 A. D. N. Vaz, S. Chakraborty and V. Massey, Biochemistry, 1995, 34, 4246.

12 C. E. French, S. Nicklin and N. C. Bruce, J. Bacteriol., 1996, 178, 6623.

13 H. Khan, R. J. Harris, T. Barna, D. H. Craig, N. C. Bruce, A. W. Munro, P. C. E. Moody and N. S. Scrutton, J. Biol. Chem., 2002, 277, 21906; P. R. Binks, C. E. French, S. Nicklin and N. C. Bruce, Appl. Environ. Microbiol., 1996, 62, 1214; C. E. French, S. Nicklin and N. C. Bruce, Appl. Environ. Microbiol., 1998, 64, 2864.

14 O. A. Blackburn, M. Tropiano, T. J. Sorenson, J. Thom, A. Beeby, L. M. Bushby, D. Parker, L. S. Natrajan and S. Faulkner, Phys. Chem. Chem. Phys., 2012, 38, 13378.

15 D. G. Smith, B. K. McMahon, R. Pal and D. Parker, Chem. Commun., 2012, 48, 8520; J. Liu, Q. Liu, C. Li, L. Sun and F. Li, J. Am. Chem. Soc., 2011, 133, 15276.

16 The change in emission over the relevant concentration range of added PETNR (0 to $60 \mu \mathrm{M}$ ) was found to be comparable for both 0.01 and 0.1 wt $\%$ UCPs. This is characteristic of ratiometric analysis.

17 H. S. Toogood, A. Fryskowska, M. Hulley, M. Sakuma, D. Mansell, G. M. Stephens, J. M. Gardiner and N. S. Scrutton, ChemBioChem, 2011, 12, 738.

18 L. J. Charbonniere and N. Hildebrandt, Eur. J. Inorg. Chem., 2008, 21, 3241. 